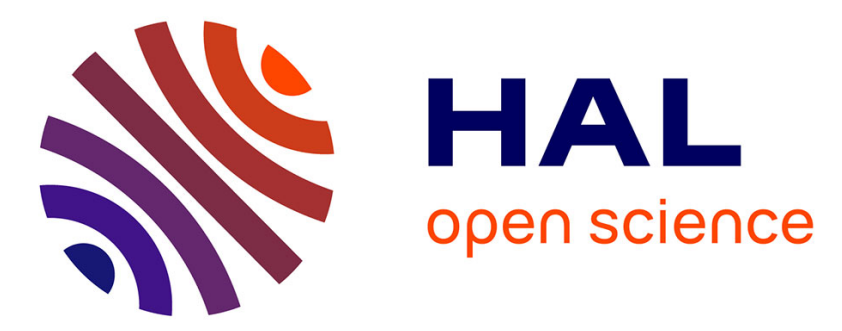

\title{
Modeling of laser ponderomotive self-focusing in plasma within the Paraxial Complex Geometrical Optics approach
}

\author{
A. Ruocco, G. Duchateau, V. Tikhonchuk, Stefan Hüller
}

\section{- To cite this version:}

A. Ruocco, G. Duchateau, V. Tikhonchuk, Stefan Hüller. Modeling of laser ponderomotive selffocusing in plasma within the Paraxial Complex Geometrical Optics approach. Plasma Physics and Controlled Fusion, 2019, Plasma Physics and Controlled Fusion, 61 (115009), 10.1088/1361$6587 / \mathrm{ab} 467 \mathrm{~b}$. hal-02289443

\section{HAL Id: hal-02289443 \\ https://hal.science/hal-02289443}

Submitted on 16 Sep 2019

HAL is a multi-disciplinary open access archive for the deposit and dissemination of scientific research documents, whether they are published or not. The documents may come from teaching and research institutions in France or abroad, or from public or private research centers.
L'archive ouverte pluridisciplinaire $\mathbf{H A L}$, est destinée au dépôt et à la diffusion de documents scientifiques de niveau recherche, publiés ou non, émanant des établissements d'enseignement et de recherche français ou étrangers, des laboratoires publics ou privés.

\section{(ㅇ)(1) $\$$}

Distributed under a Creative Commons Attribution - NonCommercial - NoDerivatives $\mid 4.0$ 


\title{
Modeling of laser ponderomotive self-focusing in plasma within the Paraxial Complex Geometrical Optics approach
}

\author{
A. Ruocco* and G. Duchateau \\ Centre Lasers Intenses et Applications (CELIA) UMR 510\%, \\ Université de Bordeaux, CNRS, CEA, 33405 Talence, France \\ V. T. Tikhonchuk \\ Centre Lasers Intenses et Applications (CELIA) UMR 5107, \\ Université de Bordeaux, CNRS, CEA, 33405 Talence, France and \\ ELI-Beamlines, Institute of Physics, Czech Academy of Sciences, \\ 25241 Dolní Břežany, Czech Republic \\ S. Hüller \\ Centre de Physique Théorique (CPHT), \\ Ecole Polytechnique, CNRS, 91128 Palaiseau, France
}




\begin{abstract}
Laser ponderomotive self-focusing in an underdense homogeneous plasma is studied within the Paraxial Complex Geometrical Optics (PCGO) approach implemented in a hydrodynamic code in 2D planar geometry. The self-focusing of a PCGO Gaussian beam is compared to simulations performed with a paraxial electromagnetic code. Good agreement has been found for beam powers less than three times the critical power and for plasma densities 5\%-10\% of the critical density. Besides Gaussian beams, PCGO allows to reproduce spatially modulated beams by superposition of Gaussian beams, mimicking a speckle pattern. Although the statistics of speckle patterns generated with PCGO reproduces well the speckle statistics of optically smoothed beams, a PCGO speckle is larger than optical speckles, carrying thus higher power such that they overestimate self-focusing effects. To overcome this issue, an algorithm is proposed within PCGO framework: it consists of superposing several Gaussian beams forming a speckle such that self-focusing effects are eventually well controlled. It is found that the superposition of three Gaussian beams with appropriate initial conditions leads to a reduction of the PCGO speckle intensity enhancement.
\end{abstract}

Keywords: laser-plasma interaction, hydrodynamics codes, beam self-focusing,inertial confinement fusion

\footnotetext{
*alessandro.ruocco@u-bordeaux.fr
} 


\section{INTRODUCTION}

Inertial Confinement Fusion (ICF) [1] is a promising way to produce clean energy. In its conventional approach, a solid capsule filled by a Deuterium-Tritium mixture is irradiated uniformly by laser beams. In order to drive a pressure of hundreds of Mbar, nanosecond laser pulses with intensities around $10^{14}-10^{15} \mathrm{~W} / \mathrm{cm}^{2}$ are needed: due to the laser-plasma coupling, the outer part of the target ablates and a plasma corona is generated. For the ICF success, laser energy must be deposited as symmetrically as possible. However, coupling between the coronal plasma and the high power lasers causes the onset of nonlinear laser-plasma interactions. Among them, ponderomotive laser beam self-focusing leads to local increasing of the laser intensity, enhancing compression nonuniformity [2]. Furthermore, local intensity enhancement may increase the probability to excite parametric instabilities, which generate hot electrons. Such electrons preheat the target, decreasing the laser compression efficiency. The use of spatial small-scale modulations of the laser intensity profile, introduced through Kinoform Phase Plates (KPP) [3], and temporal smoothing, introduced through Smoothing by Spectral Dispersion (SSD) [4], have improved the quality of laser-plasma coupling in the corona, reducing ponderomotive effects [5]. Despite that, these effects may play a role in Crossed-Beam Energy Transfer (CBET) [6], and within the Shock Ignition (SI) context [79]. The latter represents an alternative approach to the conventional ICF, where a lower intensity pulse compresses the target, and a later spike pulse launches a strong shock wave which triggers the ignition. The spike laser intensity is around $10^{16} \mathrm{~W} / \mathrm{cm}^{2}$, one order of magnitude larger than the conventional ICF.

Hydrodynamic codes are the main numerical tools to describe the processes involved in ICF since they are able to simulate appropriate spatial- and temporal-scales. However, implementation of in-line laser-plasma coupling and smoothing techniques in these codes presents a serious challenge. In the standard approach, laser energy deposition is modeled in a simplified way by using Ray-Tracing (RT) method [10]. Implementing laser speckle structure and nonlinear laser-plasma coupling processes in RT is not straightforward. In order to improve the accuracy of hydrodynamic codes through more detailed laser beam propagation models, a new method has been implemented in the hydrodynamic code CHIC [11] in two-dimensional (2D) planar geometry [12]: it relies on equations of the Paraxial Complex Geometrical Optics (PCGO) [13], an extension of the standard geometrical optics. The 
version of PCGO implemented into CHIC is called thick-ray model: along with equations for the ray trajectory, an equation for complex beam wavefront curvature is solved. This model describes propagation in plasma of beams with a Gaussian intensity profile. We refer to such beams as thick beamlets. Compared to the standard RT methods, the thick-ray model has the advantage that i) the errors in evaluation of laser absorption are reduced due to smoother distribution of deposited energy in hydrodynamic cells [12] and ii) the issue of energy deposition in caustics is partially solved thanks to the beam hasimposed a Gaussian beam shape allows to evaluate diffraction effects [13], describe the laser intensity modulations in plasma and to account for ponderomotive force [12].

The thick-ray model can be used for modeling of spatially modulated laser beams. Within this algorithm [14], several thick beamlets are created at the simulation boundaries and then propagated with random angles of incidence inside the simulation region. The uncorrelated sum of their intensity profiles creates spatially modulated intensity distribution at the focal area, mimicking the speckle pattern as in real KPP beams. The speckles generated with the thick-ray model are referred to as multi-beamlet speckles. This routine allows to reproduce the intensity statistics of real speckles and the average intensity of real smoothed beams [1416]. However, the multi-beamlet speckle structure cannot accurately model the small spatial scale of the real speckles: the superposition of thick beamlets produces larger and longer multi-beamlet speckles than the real ones. As a consequence, they carry more power than real speckles, and their self-focusing may be overestimated.

In this work, we introduce a method of controlling the speckle self-focusing by overlapping several thick rays in a speckle. Firstly, we investigate accuracy of the thick-ray model for describing beam ponderomotive self-focusing. We compare the ponderomotive self-focusing of a thick beamlet to a Gaussian-shaped beam modeled with the paraxial wave-based code HARMONY [17]. This comparison allows us to define the domain of the plasma density and beam power where the thick-ray model approximation is valid. In the second part we study the effect of self-focusing of multi-beamlet configurations which we call multibeamlet speckles. Those multi-beamlet speckles are constructed by summing the intensities of several thick beamlets which superpose inside the considered volume. The goal is to mimick the self-focusing observed in the focal region of a generally much narrower real laser speckle. For this purpose we compute the ponderomotive force acting on the plasma fluid from the superposed intensities (and not the fields) of the $N_{b}$ thick beamlets. While 
the power in each multibeamlet speckle, as in a thick beamlet, still exceeds largely the power of a narrow real speckle, the self-focusing effect on the plasma is reduced by the multibeamlet configuration with respect to the single thick beamlet. We quantify in our study the reduction effect as a function of the number $N_{b}$ of superpose beamlets and by considering two strategies for multi-beamlet speckle shaping: a random and a regular. Both approaches show that ponderomotive effects in multi-beamlet speckles are less severe than in a single thick beamlet. The regular shaping allows a better quantitative evaluation of the reduction of the ponderomotive effects.

This article is organized as follows: Section II introduces guiding theoretical considerations on beam ponderomotive self-focusing in paraxial approximation and a short description of numerical tools: the codes HARMONY and CHIC, including the PCGO model implemented in CHIC. Section III addresses propagation of a thick beamlet in plasma. A comparison between CHIC and HARMONY simulations provides a range of plasma density and beam power where the PCGO approximates in a satisfactory way the self-focusing of Gaussian beams. Section IV presents the results of self-focusing studies for the case of a multi-beamlet speckle and identification of geometrical parameters. The case of random multi-beamlet speckle shaping is considered in Section IV A, while the regular multi-beamlet speckle shaping is presented in Section IV B. Section V presents the summary of our results.

\section{THEORETICAL MODELS AND NUMERICAL TOOLS}

\section{A. Ponderomotive self-focusing in paraxial approximation. The HARMONY code}

Laser beam propagation in plasma is described within the paraxial approximation by an envelope equation for the laser electric field $\mathbf{E}[18]$

$$
\left[\frac{\partial}{\partial t}+v_{g} \frac{\partial}{\partial x}-i \frac{c^{2}}{2 \omega_{0}} \nabla_{\perp}^{2}-i \frac{\omega_{0}}{2} \frac{\delta n}{n_{c}}\right] \mathbf{E}=0 .
$$

Here $x$ corresponds to the direction of laser beam propagation, $v_{g}=c \sqrt{1-n_{e} / n_{c}}$ is the light group velocity in the plasma, $c$ and $\omega_{0}$ are the speed of light in vacuum and the laser frequency, $\delta n=n_{e}-n_{e 0}$ is the electron density perturbation with respect to the initial electron density $n_{e 0}, n_{c}=m_{e} \epsilon_{0} \omega_{0}^{2} / e^{2}$ is the critical density, $\epsilon_{0}$ is the vacuum dielectric permittivity, $e$ and $m_{e}$ are the elementary charge and electron mass, $\nabla_{\perp}^{2}=\partial_{z}^{2}+\partial_{y}^{2}$ is the 
transverse Laplacian in Cartesian coordinates, which accounts for the beam diffraction. The fourth term is responsible for beam refraction on self-induced density perturbation.

The laser electric field propagation Eq. (1) is coupled to the plasma hydrodynamic equations:

$$
\begin{gathered}
\frac{\partial n_{e}}{\partial t}+\nabla\left(n_{e} \mathbf{v}_{p}\right)=0 \\
n_{e}\left[\frac{\partial}{\partial t}+\left(\mathbf{v}_{p} \cdot \nabla\right)\right] \mathbf{v}_{p}=-\nabla P_{T}-\nabla U_{p}
\end{gathered}
$$

where $n_{e}=Z n_{i}$ is the plasma density in the quasi-neutral approximation, $m_{i}$ is the ion mass, $\mathbf{v}_{p}$ is the plasma velocity, $U_{p}=n_{e} e^{2}|E|^{2} / 4 m_{e} \omega_{0}^{2}=n_{e} /\left(2 c n_{c}\right) I$ is the ponderomotive potential, $I$ being the laser intensity, and $P_{T}=n_{i}\left(Z T_{e}+\gamma T_{i}\right)$ is the thermal plasma pressure. Here $\gamma$ represents the heat capacity ratio. All along this work, we take $\gamma=3$ which corresponds to one degree of freedom as ions move transversally to the laser propagation direction.

Solving Eqs. (2) and (3) in combination with Eq. (1), one obtains the laser field in plasma coupled to the density perturbation. When an equilibrium between the thermal and ponderomotive pressure has established, the relation between the density perturbation and the ponderomotive force reads [19]

$$
\left[\frac{|\delta n|}{n_{e 0}}\right]_{\max }=1-\exp \left(-I_{\max } / 2 c n_{c} T_{\text {eff }}\right),
$$

where $T_{\text {eff }}=T_{e}+3 T_{i} / Z$ is the plasma effective temperature, $I_{\max }$ stands for the intensity maximum due to self-focusing. For $I_{\max } \ll 2 c n_{c} T_{\text {eff }}$, the density perturbation is directly proportional to the local laser intensity, namely

$$
\left[\frac{|\delta n|}{n_{e 0}}\right]_{\max }=\frac{I_{\max }}{2 c n_{c} T_{\mathrm{eff}}} .
$$

In the case of cylindrical symmetry, the power of a Gaussian beam at each position $x$ is given by $P=\int d y d z I(z, y)=\pi w^{2} I_{0}$, where $w$ is the beam waist. The electric field is defined as $E(z, y)=E_{0}(z) \exp \left[-\left(\left(y-y_{0}\right)^{2}+\left(z-z_{0}\right)^{2}\right) / 2 w^{2}\right]$ and $I_{0}=v_{g} \epsilon_{0}\left|E_{0}\right|^{2} / 2$. The critical power for beam self-focusing $P_{c}$ is given by the expression [20-22]:

$$
P_{c}=1.86 \frac{n_{c}^{2} c^{3} T_{\mathrm{eff}}}{\omega_{0}^{2} n_{e}} \sqrt{1-\frac{n_{e}}{n_{c}}} .
$$


In the following, we restrict the study to the $2 \mathrm{D}$ planar geometry, representative of the model implemented in the hydrodynamic code CHIC with one transverse coordinate $y$. In this case, the self-focusing threshold Eq. (6) must be adapted to the 2D planar geometry. The power in $2 \mathrm{D}$ is defined as $P^{2 D}=\sqrt{\pi} I_{0} w h$, where $h$ is unit length in the third virtual dimension $y$. Then according to Eq. (6), the critical power $P_{c}^{2 D}$ in $2 \mathrm{D}$ planar geometry reads:

$$
P_{c}^{2 D}=\frac{h P_{c}}{1.86 \sqrt{\pi} w} .
$$

It is convenient to characterize the laser beam with the dimensionless power $p_{2 c}=P^{2 D} / P_{c}^{2 D}$.

From numerical point of view, electromagnetic codes, such as the two-dimensional code HARMONY, provide an accurate description of laser dynamics in plasma: they solve Eq. (1) for the electromagnetic field with a complex phase, coupled to plasma Equations (2) and (3). While HARMONY is also able to consider Brillouin backscattering, in the current context it has been used only for describing laser propagation with self-focusing. HARMONY does not impose any particular shape to the beam: although the boundary conditions correspond to a Gaussian shape, the beam profile may change during the laser propagation due to laserplasma interactions. For this reason, it serves as a reference for validation of PCGO model, which in turn imposes a Gaussian shape to a single thick beamlet, as it is explained in the next subsection.

\section{B. CHIC code and the Paraxial Complex Geometrical Optical (PCGO) model}

CHIC is a hydrodynamic code routinely used for ICF simulations. This code is based on Lagrangian formulation of Eqs. (2) and (3). Furthermore, two equations for the electron and ion temperatures that account for laser heating and energy exchange between ions and electrons are included. Plasma is treated as a single fluid, two-temperature ionized gas. An unstructured mesh includes an Artibrary Lagrangian-Eulerian (ALE) option which improves the code robustness in case of strong mesh distortions [11]. In its standard version, laser beam propagation and energy deposition are described within the Ray-Tracing method, but a new module accounts for laser beam propagation within the Paraxial Complex Geometrical Optics (PCGO). Its features are described below. For the sake of clarity, we recall here the PCGO-related language: the basic element of the thick-ray model is the thick beamlet, a 
ray with a Gaussian intensity profile and a quadratic phase front curvature. Superposition of thick beamlets intensity profiles gives a multi-beamlet speckles. The ensemble of multibeamlet speckles mimicks the laser intensity modulations at the far-field within the thick-ray model.

\section{Thick-ray model}

The thick-ray model [12] implemented in CHIC is based on the PCGO framework [13]: the laser beam has a Gaussian intensity profile, characterized by its initial power $P_{0}$ and central coordinate $\mathbf{r}_{0}$. The thick beamlet trajectory $\mathbf{r}_{0}(\tau)$ along the curvilinear ray coordinate $\tau$ obeys to the geometrical optics equation:

$$
\frac{d^{2} \mathbf{r}_{0}(\tau)}{d \tau^{2}}=-\frac{c^{2}}{2} \nabla \operatorname{Re} \epsilon
$$

where $\epsilon=1-\left(n_{e} / n_{c}\right) /\left(1-i \nu_{e i} / \omega_{0}\right)$ is the dielectric permittivity of the plasma and $\nu_{e i}$ is the electron-ion collision frequency. The beam deposes its energy in plasma according to the relation $d I / d \tau=-\omega_{0} I \operatorname{Im} \epsilon$, which accounts for electron-ion collisions (inverse Bremsstrahlung). The intensity profile $I(q, \tau)=I_{0}(\tau) \exp \left(-q^{2} / w^{2}\right)$ is related to the complex wavefront curvature $B: w(\tau)=\sqrt{2 /\left(k_{0} \operatorname{ImB}\right)}$ where $k_{0}=\omega_{0} / c$ and $q$ is the transverse coordinate normal to $\tau$. The wavefront curvature $B$ evolves along the ray trajectory according to an ordinary Riccati-type differential equation:

$$
\frac{1}{c} \frac{d B}{d \tau}+B^{2}=-\frac{3}{4 \operatorname{Re} \epsilon}\left(\frac{\partial \operatorname{Re} \epsilon}{\partial q}\right)^{2}+\frac{1}{2}\left(\frac{\partial^{2} \operatorname{Re} \epsilon}{\partial q^{2}}\right) .
$$

By solving Eqs. (8) and (9), one finds the thick beamlet intensity distribution everywhere in plasma.

\section{Superposition of thick beamlets: Multi-beamlet speckle}

While modeling of smoothed beams within PCGO, a laser beam in plasma is described as a combination of many beamlets. Each beamlet propagates according to Eqs. (8) and (9), while the plasma density and temperature profiles are provided by the plasma equations updated at each time step. The phase variation along the beamlet trajectory is not considered 
when thick beamlets are used to create a speckle pattern. Thus, the local laser intensity in plasma $I(x, y)$ is calculated as a sum of $N_{b}$ neighbor beamlets intensities $I_{j}\left(\tau_{j}, q_{j}\right)[12,14]$ :

$$
I(x, y)=\sum_{j=1}^{N_{b}} I_{j}\left(\tau_{j}, q_{j}\right),
$$

where $\tau_{j}$ is the closest position on the trajectory of the beamlet from the observation point $(x, y)$, and $q_{j}$ is the distance from $\tau_{j}$ to the observation point. Only a limited number of beamlets passing at a distance of the order of the beamlet's width contribute to the local intensity. The intensity distribution in plasma is prescribed by the focusing conditions of beamlets, which are randomly distributed in the far field. Correspondingly, the intensity distribution in plasma presents many local maxima and minima. Each local maximum represents a speckle, which is constructed by superposition of several beamlets. Hereinafter we refer to a single speckle modeled within the PCGO approach as a multi-beamlet speckle. Knowing the local intensity, one can calculate the ponderomotive pressure in plasma $U_{p}=n_{e 0} /\left(2 c n_{c}\right) \sum_{j=1}^{N_{b}} I_{j}\left(\tau_{j}, q_{j}\right)$.

Despite the thick-ray model does not account for beamets interference, the intensity statistics of the optical speckles is quite well retrived, especially for high intensity speckles [14]. However, the thick-ray approximation underestimates the amplitude of laser intensity fluctuations of a realistic spatially modulated beam. Considering the correlation length between the neighboring speckles, the contrast for a nonuniform beam at a fixed time is defined as $C^{2}=\left(\left\langle I^{2}\right\rangle-\langle I\rangle^{2}\right) /\langle I\rangle^{2}[16]$. Such a parameters quantifies the amplitude of laser intensity fluctuations. The spatial average is calculated over an area larger than the transverse beam profile. In the case of a realistic beams with a random discrete or continuous phase plates, $C=100 \%$, which means that the intensity fluctuations are comparable to the average intensity. This value cannot be retrieved within the current thick-ray approximation for two reasons: i) amplitude of intensity fluctuations in the thick-ray model is smaller than in the reality as the complex field interference is substituted by summing of scalar thick beamlets intensity profiles, and ii) diffraction limits the PCGO resolution to a few laser wavelengths. Moreover, the cell size in hydrodynamic codes is restrained to values of 5-10 $\mu \mathrm{m}$, much larger than the typical width of laser speckles of 3-5 laser wavelengths. These constraints limit the choice of the number of beamlets and beamlet parameters. The beamlet waist should be larger than the hydrodynamic cell size and there should be a few thick beamlets per speckle 
in order to maintain the contrast at a level $\sim 60-70 \%$. In this paper, we show how the PCGO-based method as thick-ray model allows to reduce the multi-beamlet intensity enhancement compared to the case of a single thick beamlet by spatial shaping of overlapping beamlets.

\section{SELF-FOCUSING OF A SINGLE THICK BEAMLET}

Propagation of a single thick beamlet in an underdense hydrogen $(Z=1)$ plasma is considered here. The thick-ray results are compared to numerical results obtained in HARMONY simulations for the same initial beam profiles. Laser absorption and plasma heating have been switched off. In this way, parametric instabilities, hot electron generation and thermal self-focusing are excluded and plasma dynamics is solely dominated by the ponderomotive force. The ponderomotive effects are evaluated by measuring the laser intensity enhancement in plasma, which is the main parameter used to compare the results of the two codes. The input parameters set for CHIC and HARMONY are such as to generate a Gaussian beam with initial beam waist $w \approx 21 \mu \mathrm{m}$, and wavelength $\lambda=1.05 \mu \mathrm{m}$. The focal plane is placed at $\approx(1100,10) \lambda$ inside a plasma of size $(2500 \times 200) \quad \lambda^{2}$ and temperature $T_{\text {eff }}=1 \mathrm{keV}$. In order to explore different regimes, two parameters have been varied: the plasma density $n_{e 0}$ and the laser power $P^{2 D}$. We have chosen: $n_{e 0} / n_{c}=0.01$; $0.05 ; 0.1$ and $p_{2 c}=1 ; 2 ; 4 ; 6$, where the value of the beam power and the critical power are recalculated for each plasma density according to Eqs. (6) and (7). The laser pulse has a step-like temporal shape lasting $t_{f}=250 \mathrm{ps}$, long enough to attain a quasi-stationary state for $t>t_{c_{s}}$ where $t_{c_{s}}=w / c_{s} \approx 70-80 \mathrm{ps}$. A quasi-steady state is achieved in case of thick-ray simulations: the thick beamlet is transversally shrinked, and its intensity slightly oscillates in position and amplitude. Quasi-stationary states were not fully attained in HARMONY simulations for large powers because of filamentation instability. According to Eqs. (4) and (5), at high densities, laser intensity enhancement is accompanied with a weak density depletion, whereas for lower densities, one expects for the same intensity increasing a stronger density depletion [22]. The CHIC results confirm this prediction: at high powers and for $n_{e 0} / n_{c}=0.05-0.01$ the plasma perturbation overcomes $10 \%$, and becomes nonlinear.

Figure 1 displays the on-axis intensity profile normalized to the initial maximum intensity $I(x) / I_{0}$ as a function of the longitudinal coordinate $x / \lambda$ for thick-ray (solid blue line) and 


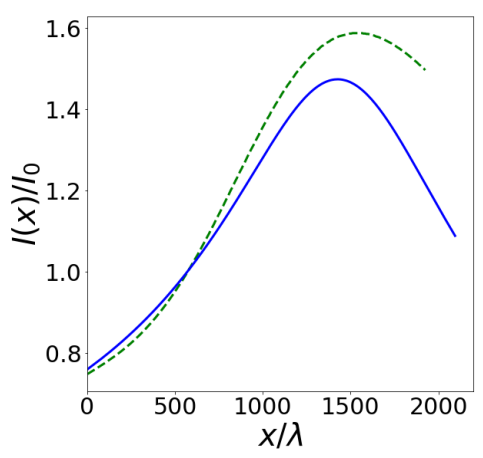

(a)

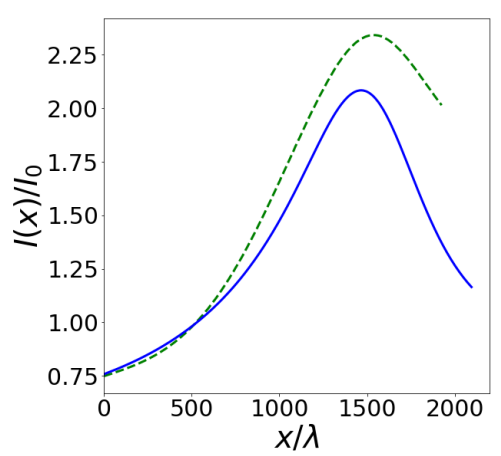

(b)

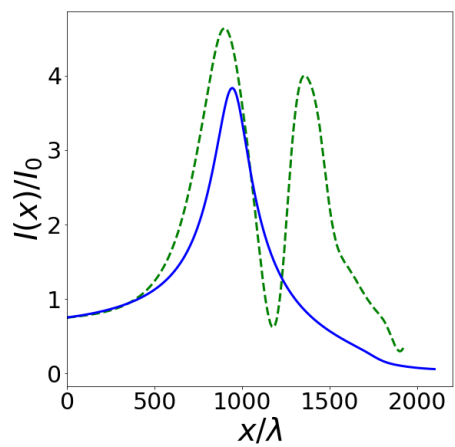

(c)

FIG. 1: On-axis intensity normalized to the initial intensity maximum $I(x) / I_{0}$ as a function of the longitudinal coordinate $x / \lambda$ at $t \approx 200 \mathrm{ps}$. The solid blue line and the dashed green curve correspond to thick-ray and HARMONY simulations respectively: (a) $n_{e 0} / n_{c}=0.1$ and $p_{2 c}=1$, (b) $n_{e 0} / n_{c}=0.05$ and $p_{2 c}=2$, (c) $n_{e 0} / n_{c}=0.01$ and $p_{2 c}=6$. The laser comes from the left side.

HARMONY (dashed green line) simulations at $t=200$ ps. Figures $1(\mathrm{a})$ and $1(\mathrm{~b})$ refer to the case $p_{2 c}=1$ and $n_{e 0} / n_{c}=0.1$, and $p_{2 c}=2$ and $n_{e 0} / n_{c}=0.05$ respectively, and show a good agreement between thick-ray and HARMONY, with a difference of less than $10 \%$ in the peak intensity and self-focusing position.

In contrast, for $p_{2 c}=6$ and $n_{e 0} / n_{c}=0.01$ of Fig. $1(\mathrm{c})$, the beam does not keep a Gaussian profile in HARMONY simulations: a second peak appears after the first main peak. The intensity evolves in time due to filamentation instability. The latter cannot be described within the thick-ray approach, and hence the on-axis profiles look considerably different. Despite that, the first peak position and its intensity are still in agreement with HARMONY within an error of $20 \%$.

Figure 2 summarizes the main results of the simulations performed: the intensity enhancement $I_{\max } / I_{0}$ is shown as a function of the beam power and for various densities. The dashed green lines represent the HARMONY results, whereas the blue lines the thick-ray results. The error bars represent the standard deviation of intensity enhancement for times $t>t_{c_{s}}$, due to intensity oscillations in time. Large error bars for the thick-ray curves at high powers are due to the that fact the beamlet waist becomes 3-4 times the laser wavelength, and the paraxial approximation becomes less accurate. For $p_{2 c} \geq 4$ and at any density, HARMONY simulations follow the theory prediction for filamentation instability [23]: strong 


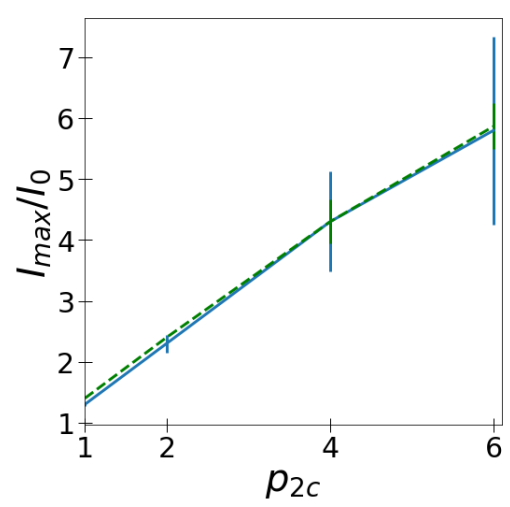

(a)

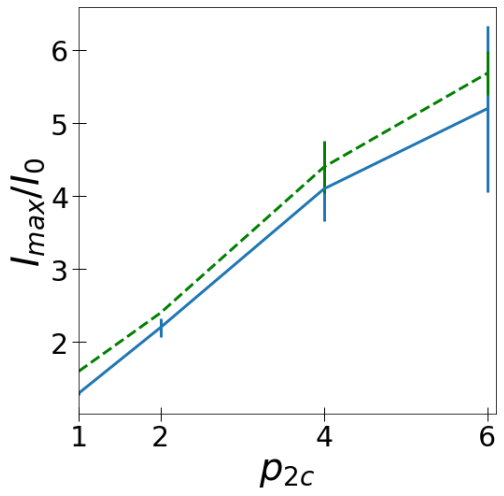

(b)

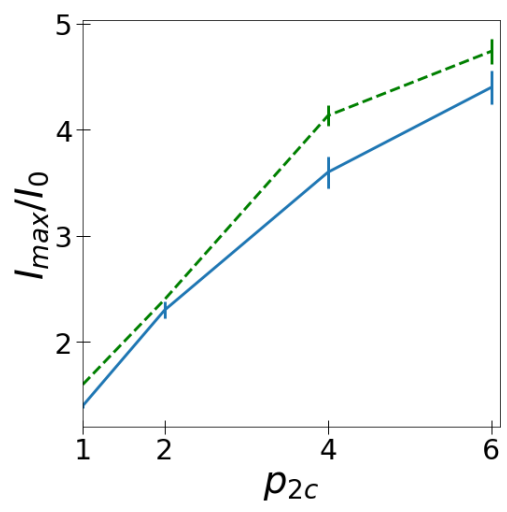

(c)

FIG. 2: Intensity enhancement $I_{\max } / I_{0}$ evaluated after the self-focusing as a function of the normalized laser beam power $p_{2 c}$ for various plasma densities: (a) $n_{e 0} / n_{c}=0.1$, (b) $n_{e 0} / n_{c}=0.05$, (c) $n_{e 0} / n_{c}=0.01$. The error bars represent the standard deviation of the time-averaged intensity enhancement. Blue and dashed green lines correspond to the thickray and HARMONY results respectively.

self-focusing leads the laser intensity to concentrate in a first peak, which afterwards breaks in filaments. The main filament carries more than $90 \%$ of the initial beam power and propagates along the same initial beam direction, whereas side filaments carry the remaining beam energy. Dashed green lines for $p_{2 c} \geq 4$ in Fig. 2 represent the intensity enhancement of the main filament. As shown in Fig. 2(a), thick-ray predictions are in good agreement for $n_{e 0} / n_{c}=0.1$. At lower density, agreement is less accurate, as one can see in Figs. 2(b)-2(c) for $n_{e 0} / n_{c}=0.05$ and $n_{e 0} / n_{c}=0.01$, with a larger difference for $n_{e 0} / n_{c}=0.01$ at higher powers because of filamentation instability. However, the difference remains within 15-20\%, which is still satisfactory.

The nature of plasma response may explain the difference at lower densities. Figure 3 shows the amplitude of density perturbations at the self-focusing position, i.e. at the position of the maximum intensity enhancement when a quasi-steady state is reached: the blue lines refer to CHIC simulations, the green lines refer to HARMONY simulations. The red and purple dashed lines stand for the theoretical estimation of the amplitude of the density response according to Eqs. (5) and (4) respectively: $I_{\max }$ is replaced by the intensity enhancement observed in CHIC simulation (see Fig. 2, blue curves). Agreement between theoretical predictions, thick-ray and HARMONY results is excellent for $n_{e 0} / n_{c}=0.1$, as 


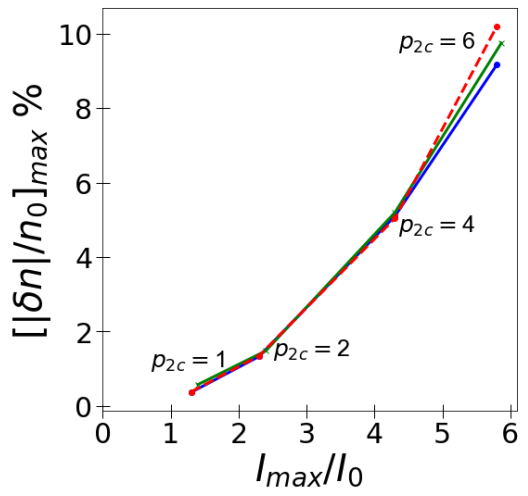

(a)

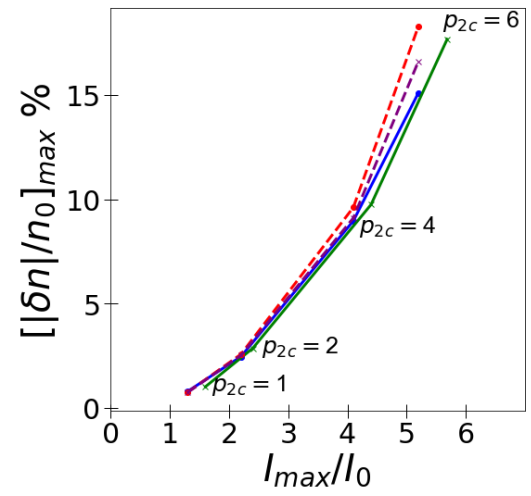

(b)

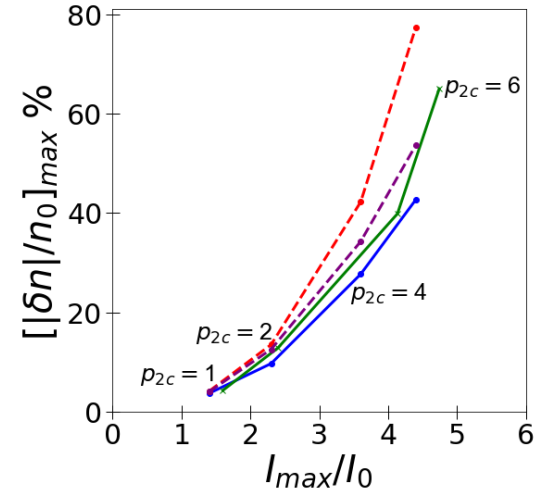

(c)

FIG. 3: Amplitude of the density perturbation $\left[|\delta n| / n_{e 0}\right]_{\max }$ as a function of laser intensity enhancement $I_{\max } / I_{0}$ for various densities: (a) $n_{e 0} / n_{c}=0.1$, (b) $n_{e 0} / n_{c}=0.05$, (c) $n_{e 0} / n_{c}=0.01$. The green and the blue lines refer to HARMONY and PCGO-CHIC results respectively, the red and purple dashed lines to the amplitude of density perturbation estimated with the thick-ray intensity amplification according to linear theory, Eq. (5), and nonlinear theory, Eq. (4), respectively.

shown in Fig. 3(a). The case $n_{e 0} / n_{c}=0.05$ is illustrated in Fig. 3(b): for $p_{2 c} \leq 2$ the agreement is still good, whereas for $p_{2 c}>2$, a shift between HARMONY and CHIC curves suggests that the laser-plasma coupling in thick-ray model fails to correctly describe the laser and plasma dynamics: the density perturbation, exceeding 10\%, becomes nonlinear. Despite the filamention instability affects the HARMONY results, the difference between the CHIC and HARMONY solutions remains less than 5-10\%. At $n_{e 0} / n_{c}=0.01$ (see Fig. 3(c)) the nonlinearity in the plasma response appears at lower power, $p_{2 c} \approx 2$ : the theoretical predictions for CHIC density response and intensity enhancement are better approximated by the nonlinear relation (purple curve), but still the simulations underestimate the density response for higher intensity enhancements. Thus, the density response in CHIC simulations results less accurate, leading to differences compared to theoretical predictions and HARMONY results. However, the difference remains lower than $20 \%$ which is still satisfactory. For $p_{c}>2$, the above theoretical considerations do not apply to HARMONY results due to filamentation instability, thus any relation among intensity enhancement and density perturbation does not hold.

We conclude this section by a discussion on the reliability of the PCGO-based thick- 
ray approach. Thick beamlets cannot break in filaments since filamentation instability is not compatible with the assumption that they must keep a Gaussian shape all along their propagation. Therefore, filamentation instability sets an upper limit for accurate description of Gaussian beam dynamics in thick-ray approximation. The full-wave description predicts filamentation instability for $p_{2 c} \geq 4$. Nevertheless, one can safely extend the thick-ray validity to $p_{2 c} \leq 6$ as only one of these filaments carries dominant part of beam energy. We also found that at low densities, the coupling between the hydrodynamics response in $\mathrm{CHIC}$ and the intensity enhancement predicted by the thick-ray model becomes less accurate because of the nonlinear plasma response. However, this issue is less important when considering a realistic density profile as encountered in ICF. Since the critical power is inversely proportional to the density, beam self-focusing is most likely to occur at high densities.

\section{SELF-FOCUSING OF A MULTI-BEAMLET SPECKLE}

The propagation of a multi-beamlet speckle is considered here. The plasma conditions are the same as in Sec. III, keeping the density fixed at $n_{e 0} / n_{c}=0.1$ and with a plasma temperature $T_{\text {eff }}=1 \mathrm{keV}$. In these conditions, the thick-ray approximation is the most accurate as possible for describing self-focusing effects. Therefore, the results shown in Section III represent the reference case for the multi-beamlet speckle study. Two methods of multi-beamlet speckle formation are compared: random and regular. In the random shaping, several thick beamlets are randomly focused in a limited area, so the speckle shape was varied in each realization. Conversely, in the regular shaping, the beamlets are focused in a way to form a speckle with a prescribed Gaussian shape. In both cases, the initial multi-beamlet speckle power is equally split over the $N_{b}$ beamlets: $P_{\text {beamlet }}=P_{\text {speckle }} / N_{b}$. The intensity enhancement in a multibeamlet speckle is compared to the intensity amplification in a single thick beamlet in order to define the method that better allows to control ponderomotive effects in a multi-beamlet speckle. 


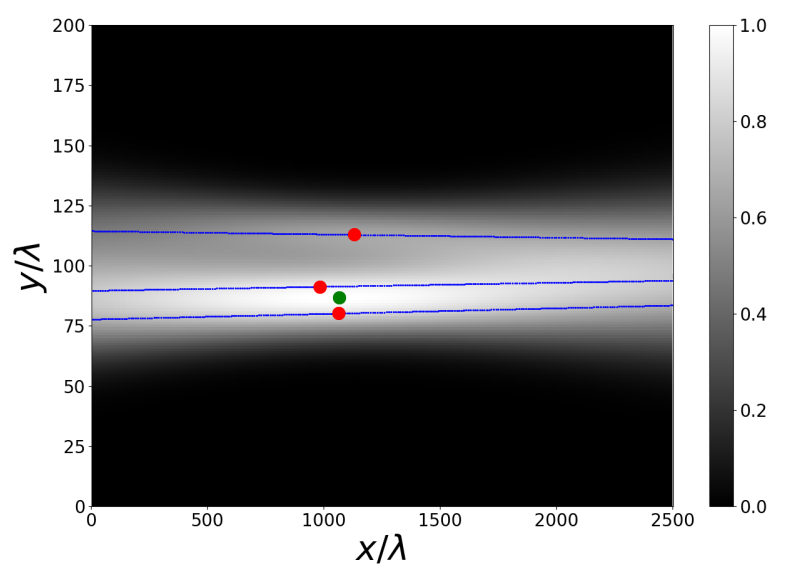

(a)

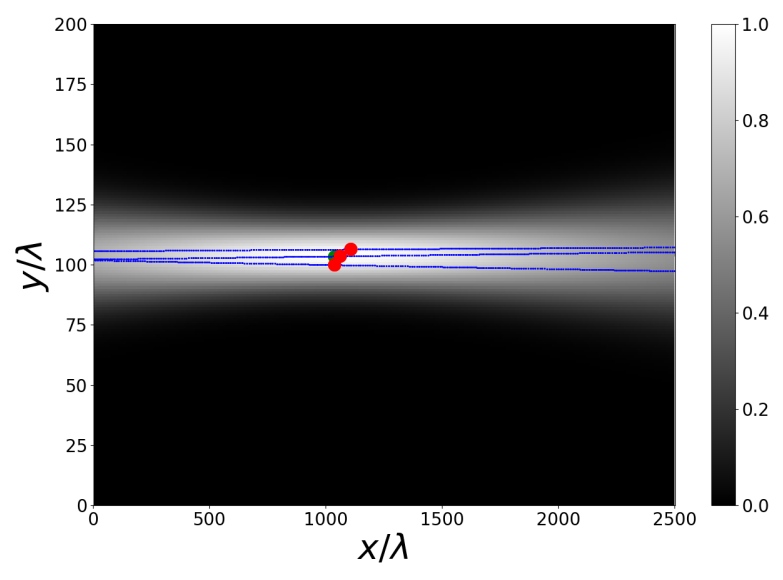

(b)

FIG. 4: Initial beamlets configuration in two different runs within the random speckle shaping method for $N_{b}=3$. The blue dashed curves show the beamlet trajectories. Addition of all beamlets intensities gives rise to multi-beamlet speckle intensity in plasma, which is indicated by the gray bar. The intensity is normalized to the maximum intensity in plasma. The red points stand for beamlet focusing points, whereas the green point for the multibeamlet speckle focus position. The laser comes from the left side.

\section{A. Multi-beamlet speckle: random speckle shaping}

Propagation of a multi-beamlet speckle via the method of random shaping is studied here. Thick beamlets, having a waist of $20 \mu \mathrm{m}$ are randomly focused in plasma in a focal zone of size $(40 \times 20) \lambda^{2}$, giving a multi-beamlet speckle focusing point located around $(1000,100)$ $\lambda$. The angles of incidence of the beamlets have been changed in each simulation, being randomly determined between $\hat{\theta}$ and $-\hat{\theta}$, where $\hat{\theta}=0.2^{\circ}$ is the averaged multi-beamlet speckle divergence. The values of thick beamlets angles of incidence and the sides of the focusing box have been chosen to reproduce a not too distorted multi-beamlet speckle, sufficiently close to a Gaussian-shaped beam. For each realization, the simulation time is $t_{f}=250 \mathrm{ps}$. In order to accumulate statistics and investigate average behavior of such a multi-beamlet speckle, we performed 5 simulations for each case for a given speckle power $P_{\text {speckle }}$. Four cases are considered: $p_{2 c}=P_{\text {speckle }} / P_{c}^{2 D}=1 ; 2 ; 4 ; 6$, where $P_{c}^{2 D}$ is evaluated according to Eq. (7). The number of beamlets $N_{b}$ is varied from 3 to 5 . The total power 


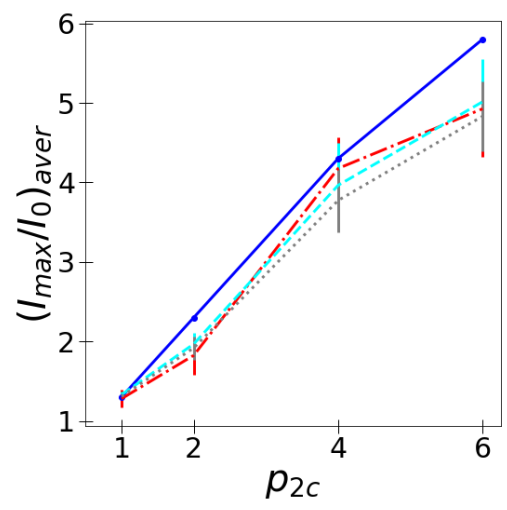

FIG. 5: Laser intensity enhancement averaged over 5 simulations $\left(I_{\text {max }} / I_{0}\right)_{\text {aver }}$ when a stationary state is reached in function of the beam power for $n_{e 0} / n_{c}=0.1$. The solid blue line refers to the single beamlet case (see Fig. 2(a)), the red dashed dotted line to $N_{b}=3$, the dashed cyan line to $N_{b}=4$ and the dotted gray line $N_{b}=5$. The error bars refer to the standard deviation of the average.

is equally split over the thick beamlets. Then, for $N_{b}=3$, each thick beamlet carries $33 \%$ of $P_{\text {speckle }}$, for $N_{b}=4$ it carries $25 \%$ of $P_{\text {speckle }}$ and for $N_{b}=5$ it carries the $20 \%$ of $P_{\text {speckle }}$. Such power splitting recreates the conditions as in multi-beamlet speckles which reproduce spatially modulated beams withing the thick-ray algorithm. The most intense speckles are composed by beamlets having the same power, which facilitates occurrence of ponderomotive effects. We remark that in this work the self-focusing of a single multi-beamlet speckle is analyzed.

Figure 4 presents an example of multi-beamlet speckle intensity distribution for $N_{b}=$ 3 with two different initial focusing configurations. It is shown how different beamlets configuration changes the multi-beamlet speckle shape. The blue dashed curves show the thick beamlets trajectories, the red points stand for beamlet focusing spots, whereas the green points for the multi-beamlet speckle focus position. Figure 5 shows the laser intensity enhancement averaged over 5 simulations $\left(I_{\max } / I_{0}\right)_{\text {aver }}$. The solid blue line refers to the single beamlet intensity amplification described in Sec. III (see Fig. 2(a)), other lines refer to multi-beamlet cases: the red dashed dotted line refers to the case $N_{b}=3$; the dashed cyan line to the case $N_{b}=4$ and the gray dotted line to the case $N_{b}=5$. The intensity enhancement in a multi-beamlet speckle is reduced with respect to the single beamlet case: 
the maximum reduction is $15 \%$ for high power $\left(p_{2 c}=6\right)$, with a weak dependence on $N_{b}$ for $p_{2 c} \geq 2$. Since the thick beamlets carry a fraction of the total speckle power, the speckleplasma dynamics is decoupled: the local plasma density perturbation is less deep and more spread along the density channel compared to the single beamlet case presented in Sec. III. Thus, randomly superposed thick beamlets reduce the intensity amplification compared to a single beamlet with the same power. The reduction however cannot be deterministically quantified from the initial beamlets configuration, since the multi-beamlet speckle profile is not a priori defined. Results not reported here for the sake of conciseness show that increasing the number of beamlets beyond $N_{b}=5$, the intensity enhancement for such multi-beamlet speckles follows the same tendency as for $N_{b} \leq 5$, demonstrating that ponderomotive effects weakly depends on $N_{b}$ within the random shaping method. Also, simulations performed with different power splitting show that if one beamlet carries more than $50 \%$ of the total power, it governs the multi-beamlet speckle self-focusing similarly to the single beamlet case. However, unequal distribution of the speckle power over the beamlets is not compatible with the PCGO algorithm developed for multi-speckle beams.

\section{B. Multi-beamlet speckle: regular speckle shaping}

In this section we study a multi-beamlet speckle containing 3 and 4 regularly focused beamlets. This choice is motivated by the results of the previous section: the self-focusing depends weakly on the number of beamlets. We present the most favorable case for selffocusing controlling of three superposed beamlets in Fig. 6(a). We show also another case of not-so-favorable configuration of four superposed beamlets in Fig. 6(b). In case $N_{b}=3$ the focus point of the speckle is located at $(1000,100) \lambda$, the green point: the edge beamlets enter the plasma with angles of incidence of $\pm 0.02^{\circ}$ with respect to the central beamlet which in turns propagates parallel to the $x$ direction. Their focusing positions (red points) are placed at: $(900,110) \lambda,(1100,100) \lambda$ and $(1300,90) \lambda$ respectively. For $N_{b}=4$, in Fig. $6(\mathrm{~b})$, the two central thick beamlets are focused at $(800,100) \lambda$ with an angle of incidence of $\pm 0.01^{\circ}$ respectively, whilst the edge thick beamlets are focused at $(1200,110) \lambda$ and $(1200,90) \lambda$ with an angle of incidence of $\pm 0.02^{\circ}$. The transverse and longitudinal profiles for each case

are shown in Fig. 7. The solid blue lines stand for a reference Gaussian profile, whereas the dashed dotted red line and the dashed cyan line for $N_{b}=3$ and $N_{b}=4$ respectively. 
Figure 7(a) refers to the transverse profile at the focus position, whereas Fig. 7(b) refers to the longitudinal profile. The matching with the Gaussian longitudinal and transversal profiles is good in all cases. Figure 8(a) shows the results of intensity enhancement in the speckle formed with three beamlets compared to single beamlet simulations presented in Sec. III (solid blue line in Fig. 2(a)). The intensity enhancement $I_{\max } / I_{0}$ is taken in the quasi-stationary state. The error bars refer to the standard deviation of the time-averaged intensity enhancement since the maximum peak intensity varies slightly in position and amplitude. The dashed red line refers to the case where the multi-beamlet speckle power is set equal to single beamlet cases (see Sec. III), so $p_{2 d}=P_{\text {speckle }} / P_{c}^{2 D}=P_{2 D} / P_{c}^{2 D}$, where $P_{2 D}$ is the power of a single thick beamlet as defined in Sec. III. The laser intensity enhancement is reduced in multi-beamlet speckle. Similarly to the case of random multi-beamlet, since the thick beamlets do not fully overlap, they create a density channel along the speckle propagation axis longer than the single beamlet case. Such a density channel is less deep compared to the case of a single beamlet, leading to a weaker self-focusing. Despite that,

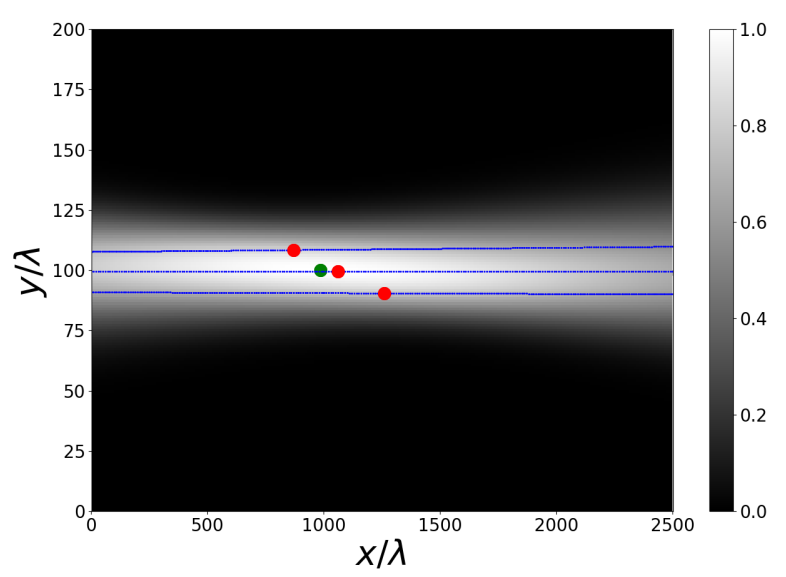

(a)

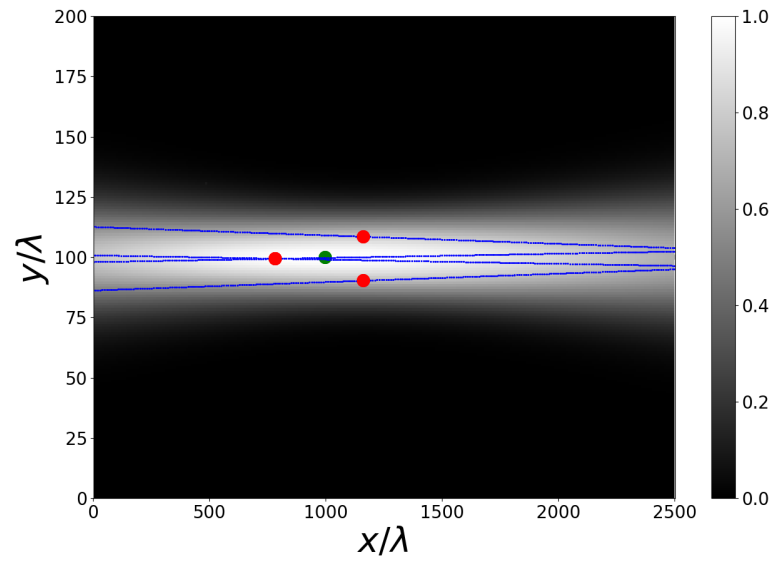

(b)

FIG. 6: Example of regular multi-beamlet speckle shaping for $N_{b}=3$ (a) and $N_{b}=4$ (b). The blue dashed curves show the thick beamlets trajectories. Addition of all thick beamlets intensities gives rise to multi-beamlet speckle intensity indicated by the gray bar. The intensity is normalized to the maximum intensity in plasma. The green points stand for the multi-beamlet speckle focus position, whereas the red points stand for the thick beamlet focusing points. The laser comes from the left side. 


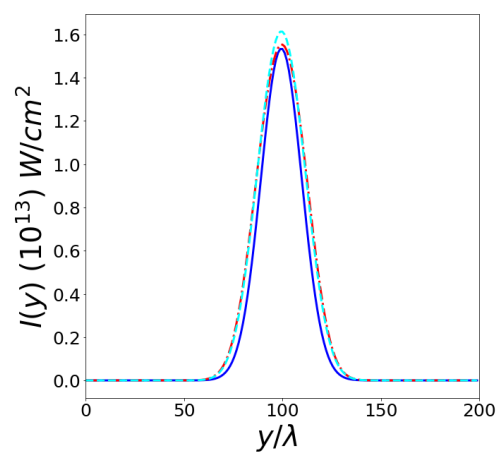

(a)

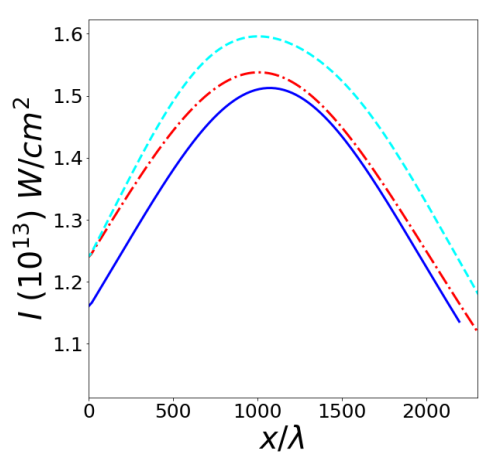

(b)

FIG. 7: Laser intensity transverse cut in the focal plane (a) and longitudinal laser intensity distribution along the beam propagation direction (b) for the single beamlet (solid blue line) and a regularly shaped multi-beamlet speckle for $N_{b}=3$ (red dashed dotted line) and $N_{b}=4$ (dashed cyan line). In (b), the laser enters from the left. In both pictures, $p_{2 c}=1$.

the density channel is sufficiently deep to refract the beamlets trajectories and guide them along the channel axis, resulting in spraying of thick beamlets and breaking of the initial speckle shape. All these effects contribute to self-focusing suppression compared to the case of a single thick beamlet carrying the same amount of power.

Speckle self-focusing leads to a modification of the density channel profile. Evaluation of such variations can be used for characterizing the ponderomotive effects. In case of a single Gaussian beamlet, one can define an aspect ratio as $z_{R} \lambda /\left(w^{2} \pi\right) \approx 1$, which is preserved during the beamlet dynamics. (Here $z_{R}$ is the longitudinal beamlet length.) In analogy to Gaussian beams, the longitudinal length of regularly shaped multi-beamlet speckle $z_{R}^{m b s}$ is defined as a distance from the speckle intensity maximum to where the intensity decreases by a factor of $\sqrt{2}$. At early time, before self-focusing develops, the multi-beamlet speckle has a quasi-Gaussian profile (see Figs. $6(\mathrm{a})$ and 7 ), and $\left[z_{R}^{m b s} \lambda /\left(w^{2} \pi\right)\right]_{t=0} \approx 1$. However, this aspect ratio changes in time because of ponderomotive effects locally exercised by the beamlets, and it oscillates between 1.5 and 2.6: thus, the self-focusing zone is in average around 2 times bigger than the single beamlet case. This change of the speckle shape explains reduction of the intensity enhancement in the speckle. Increase of the aspect ratio in self-focused speckle is illustrated in Fig. 9. The intensity contours with black lines correspond to plots for single beamlet self-focusing with $p_{2 c}=4$. The red and green dashed lines corresponds to 3-beamlet speckle self-focusing having the same power as the single beamlet in Fig. 9(a), 
and a power twice bigger in Fig. 9(b) respectively. The aspect ratio of single beamlet remains constant, equal to the initial value, i.e. approximatively one. Considering for the black lines that the beam width is $5 \lambda$ and that the beamlet intensity enhancement is 4.5 (see Fig. 2(a)), the longitudinal length where intensity decreases by a factor $\sqrt{2}$ is $\approx 100 \lambda$, which gives an aspect ratio $\sim 1.12$. Calculating the same aspect ratio for the 3-beamlet speckle in Fig. 9(a), one obtains around 1.7. Evaluating the aspect ratio for a speckle with a power twice higher (Fig. 9(b)), one finds an aspect ratio of around 1.3. Consequently, in order to obtain approximatively the same self-focusing area of a single beamlet, the power of the multi-beamlet speckle must be twice the single beamlet power. One can account for this by redefining the critical power for such a 3 -beamlet speckle $\tilde{P}_{c, \text { speckle }}$ as $\tilde{P}_{c, \text { speckle }}=\eta P_{c}^{2 D}$, where $\eta=2$ corresponds to the aspect ratio of the multi-beamlet speckle having the same power as the single beamlet. Rerunning the simulations for a 3-beamlet speckle with power as $p_{2 c}=P_{\text {speckle }} / \tilde{P}_{c, \text { speckle }}$, one obtains the dashed orange line in Fig. 8(a), where the intensity enhancement of the multi-beamlet speckle becomes comparable to the single beamlet

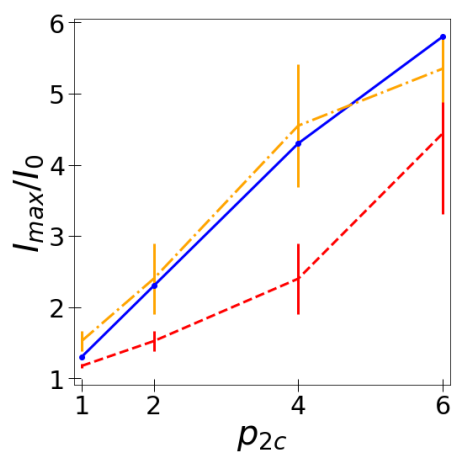

(a)

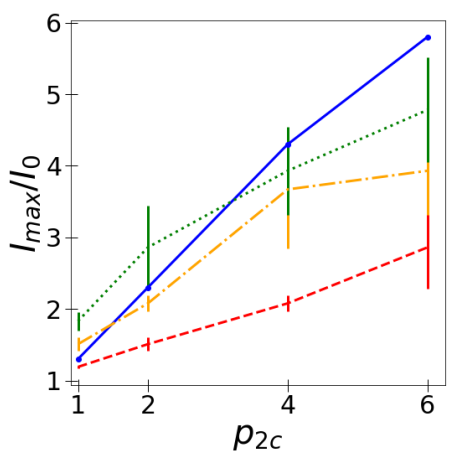

(b)

FIG. 8: Laser intensity enhancement $I_{\max } / I_{0}$ as a function of the beam power. Comparison between the single beamlet and speckle with regular shaping for $N_{b}=3$ (a) and $N_{b}=4$ (b). The plasma density is $n_{e 0} / n_{c}=0.1$. The solid blue curves refer to single beamlet results (solid blue curve in Fig. 2(a)). The red dashed lines refer to the case where the multibeamlet speckle critical power is defined according to Eq. (7), the orange dashed dotted lines show the intensity enhancement assuming that the speckle critical power is twice the value defined by Eq. (7), the dotted green line refers to the case where the critical power is multiplied by a factor of 3 . 


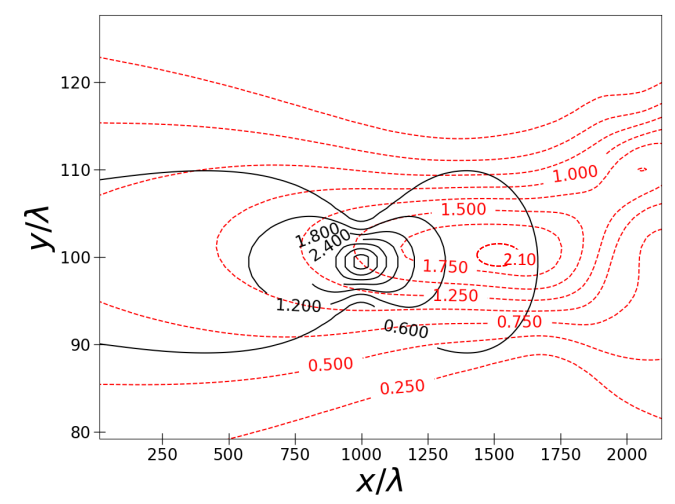

(a)

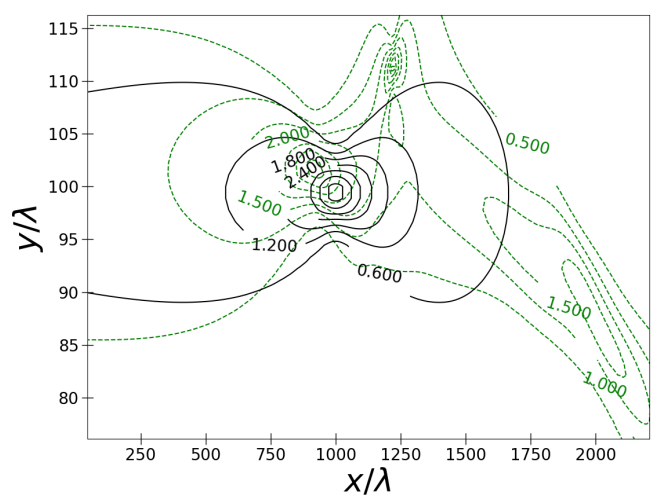

(b)

FIG. 9: Laser intensity contour plot in plasma at time 200 ps. In both pictures, the solid black lines refer to the case of single beamlet with $p_{2 c}=4$ at $t=200 \mathrm{ps}$. The red dashed lines in (a) refer to the speckle with $N_{b}=3$ having same power as the single beamlet. The green dashed line in (b) corresponds to a 3-beamlet speckle with power $\eta=2$ times bigger. The intensity is normalized to the initial maximum intensity in plasma. The laser comes from the left side.

case. Thus multiplying the speckle critical power by $\eta$ allows to retrieve the single beamlet results in terms of intensity amplification. Or, from a different point of view, one can state that superposing 3 beamlets with such the configuration as in Fig. 6(a) leads to a reduction of self-focusing effects roughly quantified by the increasing of the critical power by a factor $\eta=2$. Besides the intensity amplification in plasma, also the speckle shape around the selffocusing position is very similar to the single beamlet, as one can see in Fig. 9(b). However, despite the rescaling of critical power of the 3-beamlet speckle allows to retrieve the intensity enhancement in the plasma, several features of the standard Gaussian beam propagation are lost. This is still evidenced in Fig. 9(b): the self-focusing position of the 3-beamlet speckle, located around $(900,100) \lambda$, is shifted from the position of the single beamlet case, and deviated from the initial multi-beamlet speckle propagation axis. The 3-beamlet speckle shape is modified behind it because of beamlets refraction in the density channel. The beamlets trajectories are deviated, creating other local intensity maxima, as the one at the position $(1200,112) \lambda$. This process resembles beam spraying from a qualitatively point of view, but with a prescribed number of filaments approximatively equal to the number of beamlets. 
Figure 8(b) shows the results for a 4-beamlet speckle with an initial beamlets configuration as in Fig. 6(b): displayed is the intensity enhancement $I_{m a x} / I_{0}$ as a function of a 4-beamlet speckle power defined as $p_{2 c}=P_{\text {speckle }} / P_{c}^{2 D}$ (red dashed line), compared to the single beamlet (solid blue line). Superposition of four beamlets decreases the intensity enhancement by $50 \%$ in average. These results, similarly to the previous case could be reduced to the single beamlet case by introducing a multiplicative factor $\eta \approx 2-3$ in the critical speckle power, still related to the increasing of the self-focusing area. In Fig. 8(b), the orange dashed dotted line and the green dotted line correspond to the case for which

$p_{2 c}=P_{\text {speckle }} / \tilde{P}_{c, \text { speckle }}$, with $\eta=2$ and $\eta=3$ respectively. However, the agreement is satisfactory for $\eta=2$ for $p_{2 d}<4$. Instead, a larger discrepancy occurs for $\eta=3$ at $p_{2 d}>4$. This simplified reduction does not work at higher power. It means that the considerations made for the three beamlets speckle does not completely hold for the case $N_{b}=4$, showing that the process is strongly related to the initial beamlet configuration.

We conclude this section by commenting the results obtained in case of regularly shaped multi-beamlet speckle. Despite the reduction of ponderomotive effects is quite well attained through superposition of three and four thick beamlets, quantifying such a reduction by simply introducing a scaling factor in the multi-beamlet speckle power is not always satisfactory. The case $N_{b}=3$ shows that the ponderomotive effects are reduced, and such reduction can be accounted for by rescaling the 3-beamlet speckle critical power of a factor $\eta=2$. The value of this factor is partially justified by the speckle aspect ratio defined above. For $N_{b}=4$, one still obtains less important ponderomotive effects, which cannot be easily related to the aspect ratio. This is due by the fact that the reduction of self-focusing actually depends on several parameters, as the initial beamlets focusing positions and initial angles of incidence. Moreover, the dynamics is affected by complicated behavior of the beamlets when ponderomotive effects occur. The factor $\eta$ stands as a rough approximation of all such effects, which gives an accetable agreement only in a limited number of cases.

\section{CONCLUSION}

We have studied ponderomotive self-focusing of a laser beam in plasma within the PCGObased approach implemented in the hydrodynamic code CHIC, the thick-ray model. The validity domain of the thick-ray approximation is defined by comparison to the paraxial 
wave-based code HARMONY while simulating self-focusing of a Gaussian beam. A good agreement is found for beam powers lower than 4 times the critical power. Above that, beams undergo filamentation instability and then break up in filaments. Such a phenomenon cannot occur within the thick-ray approximation due to geometrical optics limitation. However, thick-ray simulations retrieve the laser intensity enhancement up to 6 times the critical power when comparing to the intensity enhancement of the main filament of HARMONY simulations. This assessment is confirmed for plasma density range $n_{e 0} / n_{c}=0.01-0.1$ and temperature $1 \mathrm{keV}$ pertinent to ICF conditions in corona target. A better agreement between the thick-ray approximation and HARMONY results has been found for $n_{e 0} / n_{c}=0.1-0.05$ at which density depletion is less important.

In order to approach the situation of multiple speckles in an optically smoothed laser beam, superposition of a limited number $N_{b}$ of thick beamlets created within the thickray model has been considered in order to study the self-focusing of a single multi-beamlet speckle. The goal was to overcome the fact that thick beamlets and multi-beamlet speckles are larger than real speckles. So they carry a much higher power than real laser speckles, and therefore self-focusing is overestimated. To correct the unrealistic self-focusing in a multi-beamlet speckle, two methods of speckle shaping have been presented: random and regular configurations with $N_{b}=3-5$ thick beamlets. Their superposition in intensity, with a weight (here of $1 / N_{b}$ ) for each of them, applied when computing the ponderomotive force, results in a reduced self-focusing effects with respect to a single thick beamlet carrying the same power. In both cases, reduction of intensity enhancement has been evidenced. For the case of regular multi-beamlet shaping, this reduction can be quantitatively accounted for by considering an effective increase of the critical power by a factor $\eta$ compared to the critical power of a Gaussian beam of the same width. This factor is approximately equal to the aspect ratio of the self-focused multi-beamlet speckle. In case of $N_{b}=3, \eta \simeq 2$, and for $N_{b}=4, \eta \simeq 2-3$. The validity of this approximation is limited to the case when the multibeamlet speckle power remains few times the self-focusing critical power, and, furthermore, when the multi-beamlet configuration corresponds to the initial beamlet focusing positions and the beamlets angles of incidence illustrated in Sec. IV B. Employing this method to control intensity amplification, one will be able to approximate self-focusing of a real laser speckle by superposing few beamlets with the configurations presented in this paper in 2D planar geometry. In future work, the thick-ray model in CHIC will be adapted to such 
a regular multi-beamlet method, and self-focusing of spatially smoothed beams modelled through thick-ray will be investigated.

\section{ACKNOWLEDGMENTS}

This work has been carried out within the framework of the EUROfusion Consortium and has received funding from the Euratom research and training programme 2014-2018 and 2019-2020 under grant agreement No 633053. The views and opinions expressed herein do not necessarily reflect those of the European Commission. One of the author, A.R., acknowledges J. Trela, A. Colaïtis for their suggestions on the CHIC code, and A. Maximov and V. Goncharov for useful discussions. This work was partially supported by the project ELITAS (ELI Tools for Advanced Simulation) CZ.02.1.01/0.0/0.0/16 $13 / 0001793$ from the European Regional Development Fund.

[1] S. Atzeni, J. Meyer-ter-Vehn, The physics of Inertial Fusion, Oxford University Press Canada, 2009 .

[2] R. S. Craxton, K. S. Anderson, T. R. Boehly, V. N. Goncharov, D. R. Harding, J. P. Knauer, R. L. McCrory, P. W. McKenty, D. D. Meyerhofer, J. F. Myatt, et al., Phys. Plasmas 22, 110501 (2015); doi.org/10.1063/1.4934714.

[3] Y. Kato, K. Mima, N. Miyanaga, S. Arinaga, Y. Kitagawa, M. Nakatsuka, and C. Yamanaka, Phys. Rev. Lett. 53, 1057 (1984); doi.org/10.1103/PhysRevLett.53.1057.

[4] S. Skupsky, R. W. Short, T. Kessler, R. S. Craxton, S. Letzring, and J. M. Soures, J. Appl. Phys. 66, 3456 (1989); doi.org/10.1063/1.344101.

[5] E. A. Williams, Phys. Plasmas 13056310 (2006); doi.org/10.1063/1.2179051.

[6] G. Raj and S. Hüller, Phys. Rev. Lett. 118, 055002 (2017); doi.org/10.1103/PhysRevLett. 118.055002.

[7] R. Betti, C. D. Zhou, K. S. Anderson, L. J. Perkins, W. Theobald, and A. A. Solodov, Phys. Rev. Lett. 98, 155001 (2007); doi.org/10.1103/PhysRevLett.98.155001.

[8] S. Atzeni, X. Ribeyre, G. Schurtz, A.J. Schmitt, B. Canaud, R. Betti and L.J. Perkins, Nucl. Fusion 54054008 (2014); doi.org/10.1088/0029-5515/54/5/054008. 
[9] D. Batani, S. Baton, A. Casner, S. Depierreux, M. Hohenberger, O. Klimo, M. Koenig, C. Labaune, X. Ribeyre, C. Rousseaux, G. Schurtz, W. Theobald and V.T. Tikhonchuk, Nucl. Fusion 54, 054009 (2014); doi.org/10.1088/0029-5515/54/5/054009.

[10] T.B. Kaiser, Phys. Rev. E, 891 (2000); doi.org/10.1103/PhysRevE.61.895.

[11] J. Breil, S.Galera and P.H. Maire, Computers Fluids 46, 161-167 (2011); doi:10.1016/J . COMPFLUID . 2010.06.017.

[12] A. Colaïtis, G. Duchateau, Ph. Nicolaï and V. Tikhonchuk, Phys. Rev. E 89, 033101 (2014); doi.org/10.1103/PhysRevE.89.033101.

[13] Y. A. Kravtsov, Y. I Orlov, Geometrical Optics of Inhomogeneous Media, Springer-Verlag, (1990).

[14] A. Colaïtis, G. Duchateau, X. Ribeyre and V. Tikhonchuk, Phys. Rev. E 91, 013102 (2015); doi.org/10.1103/PhysRevE.89.033101.

[15] H. A. Rose and D. F. Dubois, Phys. Fluids B 5, 590 (1992); doi.org/10.1063/1.860545.

[16] J. Garnier, Phys. Plasmas 6, 1601 (1999); doi.org/10.1063/1.873413.

[17] S. Hüller, P. E. Masson-Laborde, D. Pesme, M. Casanova, F. Detering and A. Maximov, Phys. Plasmas 13, 022703 (2006); doi.org/10.1063/1.2168403.

[18] W. Kruer, The Physics Of Laser Plasma Interactions, CRC Press, 2003.

[19] P. Kaw, G. Schmidt, and T. Wilcox, Physics of Fluids 16, 1522 (1973); doi.org/10.1063/1. 1694552.

[20] C.E. Max, Physics of Fluids 19, 74 (1976); doi.org/10.1063/1.861305.

[21] H. A. Rose and D. F. DuBois, Physics of Fluids B 5, 3337 (1993); doi.org/10.1063/1. 860629.

[22] V. Tikhonchuk, S. Hüller, P. Mounaix, Phys. Plasmas 4, 4369 (1997); doi.org/10.1063/1. 872599.

[23] E. M. Epperlein, Phys. Rev. Lett. 65, 2145 (1990); doi.org/10.1103/PhysRevLett.65.2145. 